\title{
Endometrium-limited Metastasis From Colon Carcinoma
} Irene Pecorella ${ }^{1, *}$, Chiara Di Tucci ${ }^{2}$, Emma Rullo ${ }^{1}$, Hiba Wazeer Al Zou'bi', Ludovico Muzii

${ }^{1}$ Department of Radiologic, Oncologic and Anatomic Pathology, University of Rome "La Sapienza", Viale Regina Elena, Rome, Italy

${ }^{2}$ Department of Maternity and Urological Sciences, University of Rome "La Sapienza", Viale Regina Elena, Rome, Italy

*Corresponding author: Irene Pecorella, Department of Radiologic, Oncologic and Anatomic Pathology, University of Rome "La Sapienza", Viale Regina Elena 324 - 00161, Rome, Italy. Tel: +3964997561; Fax: +39649973376; E-mail:

DOI: $10.30699 / \mathrm{mci} .5 .3 .508-1$ irene.pecorella@uniroma1.it

Submitted: 16 March 2021

Revised: 9 June 2021

Accepted: 10 July 2021

e-Published: 28 August 2021

Keywords:

Uterus

Neoplasm Metastasis

Endometrium

Colonic Neoplasms
Introduction: Extragenital tumors uncommonly affect the female genital tract and usually involve the ovaries (75-80\%), while the uterus can harbor metastases in the context of a widely disseminated disease. The primary tumors are most often located in the breast $(35 \%)$ or gastrointestinal tract $(38 \%)$. Metastases to the uterine body usually involve the myometrium whereas those exclusively involving the endometrium are rare.

Case presentation: We report the case of a 50-year-old woman with endometrial metastasis from a colonic adenocarcinoma 6 years after the initial presentation.

Conclusion: Metastatic colon tumors can histologically present as a primary disease in the endometrium if the pathologist has little awareness to consider metastasis. The clinical history of disseminated metastases, the lack of CK 7 expression at immunohistochemistry, and positive results for $\mathrm{CK} 20$ and nuclear CDX2 are helpful clues to support the diagnosis of metastatic carcinoma of colorectal origin.

(C) 2021. Multidisciplinary Cancer Investigation

\section{INTRODUCTION}

Metastases from extragenital tumors to the female genital tract are uncommon and usually affect the ovaries $(75-80 \%)$. The uterus is rarely involved, usually in the context of a widely disseminated disease. The primary tumors are most often located in the breast $(35 \%)$ or gastrointestinal tract $(38 \%)$ $[1,2]$. Metastatic tumors to the uterine body usually involve the myometrium whereas those exclusively involving the endometrium are rare. Herein, we report a case of endometrial metastasis from a colonic adenocarcinoma with a review of the literature.

\section{CASE PRESENTATION}

A 50- year-old woman underwent the right hemicolectomy in 2009 for colonic adenocarcinoma stage pT3 pN2 (3/14). Her previous medical history was notable only for a left salpingo- oophorectomy performed for an endometriosis cyst 15 years earlier. She subsequently received adjuvant chemotherapy according to FOLFOXIRI BEVA protocol (Fluorouracil, Leucovorin, Oxaliplatin, and Irinotecan plus Bevacizumab) as the treatment strategy for colon cancer, until October 2010. Her treatment was followed by two selective internal radiation therapy treatments (SIRT). In February 2013, after a disease-free interval of 20 months, the disease progressed to the involvement of the common iliac and right external iliac lymph nodes. The patient was subjected to second-line chemotherapy according to the scheme FOLFIRI (folinic acid, fluorouracil (5FU), and irinotecan) cetuximab until September 2014, followed by STEREOBODY RT from November to December 2014, for the involvement of the right iliac vein. In February 2015, total body CT 
scans revealed reduction of the disease in the lymph nodes, but also the presence of a solid tissue of about $30 \times 45 \mathrm{~mm}$ in the uterus, with peripheral vascular enhancement; obliterating the endometrial cavity. This neoformation was confirmed by a transvaginal ultrasound examination. Operative hysteroscopy with endometrial curettage was carried out in April 2015 and the material was sent for microscopical examination. Histologically, the endometrial sampling showed multiple fragments of normal endometrial tissue mixed with irregular glands lined by atypical cells with elongated and hyperchromatic nuclei. The tumoral tissue was immunoreactive for CDX2, indicating either the gastrointestinal origin or an endocervical adenocarcinoma of intestinal type. PET/ CT which was performed in May 2015 confirmed hyperfixation tracer (SUV 7.4) in the uterus and the right external iliac vessels. The patient underwent radical hysterectomy TYPE $\mathrm{B}$ with peritoneal washing and right salpingo-oophorectomy in July 2015. Histologically, an intestinal-type malignant growth was present in the endometrial layer (Figure 1A), showing intraluminal glandular dirty necrosis and moderate cytoarchitectural dysplasia (Figure 1B). The tumor cells strongly expressed cytoplasmic cytokeratin 20, nuclear CDX2, cytoplasmic p16, and carcinoembryonic antigen (CEA) and were negative for cytokeratin 7, estrogen receptor (ER) (Figure 2), Ca-125, and vimentin. Microscopical features were suggesting a colorectal etiology. The myometrium, the uterine serosa, and the peritoneal washing were free of tumor cells.

\section{DISCUSSION}

Colonic adenocarcinoma initially spreads intramurally in the intestinal wall, with involvement of the adjacent structures occurring at later stages of the disease. Visualization of the adjacent invading tumor on imaging allows for a correct diagnosis of tumor extension. Distant metastases through the blood and lymphatic stream mostly occur in the liver and lung. Metastases to the female genital tract, other than the ovaries, are a rare event, considering the centrifugal drainage of lymphatics from the uterus and the fibrous nature of the cervical stroma [3]. Therefore, colon tumors generally spread to the uterus by direct extension or widespread peritoneal implants. Noncontiguous, hematogenous, or lymphatic spread is exceptional among cases in which a widely disseminated disease is not apparent [4].

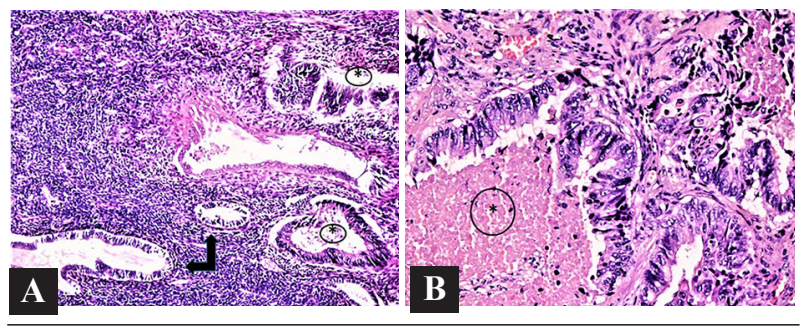

Figure 1: Intestinal-type Malignant Growth in the Endometrium A) Photomicrograph showing neoplastic glands (asterisks) within the endometrium. Residual normal endometrial glands can be noticed (double arrow); B) Higher magnification of the large bowel cancer, showing the characteristics dirty necrosis (asterisk).

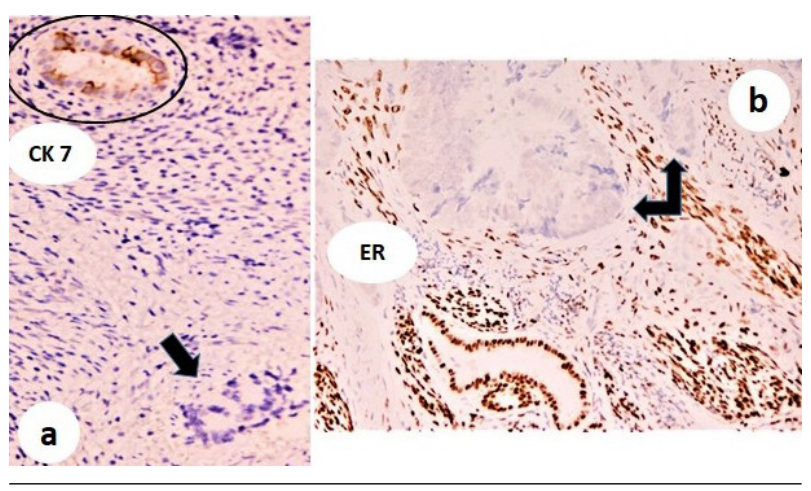

Figure 2: Immunohistochemical Staining of the Normal Endometrial Glands and the Metastatic Colon Carcinoma A) Immunohistochemistry shows positive results for cytokeratin 7 in an endometrial gland (upper part) and negative results in the infiltrating colon cancer (arrowhead); B) The neoplastic cells (double arrow) also fail to immunostain with an antioestrogen receptor antibody. ER+ nuclei of endometrial glands and myometrial cells are seen in the background.

Even so, metastatic deposits occur more frequently in the myometrial layer than in the endometrial mucosa1. Isolated metastasis of a large bowel adenocarcinoma (including rectal carcinoma) to the endometrium is generally considered an extremely rare event. Kumar et al., [1] detected 11 colon cancers metastatic to the uterine body in their review of 63 extragenital cancers causing uterine metastases (17.5\%). However, the majority (63.5\%) of the metastases from extragenital cancers involved the myometrium and only $3.8 \%$ of those cases were limited to the endometrium [1]. An incidence of $3.6 \%$ endometrial metastasis was reported by Mazur et al., in a series of 56 colorectal cancers metastatic to the female genital tract [2]; confirming the figure reported by Kumar et al., [1]. It should be noted that most cases of myometrial involvement are associated with synchronous ovarian metastasis [5], so that they may represent tumor spread from exfoliated ovarian tumor cells. In such instances, 


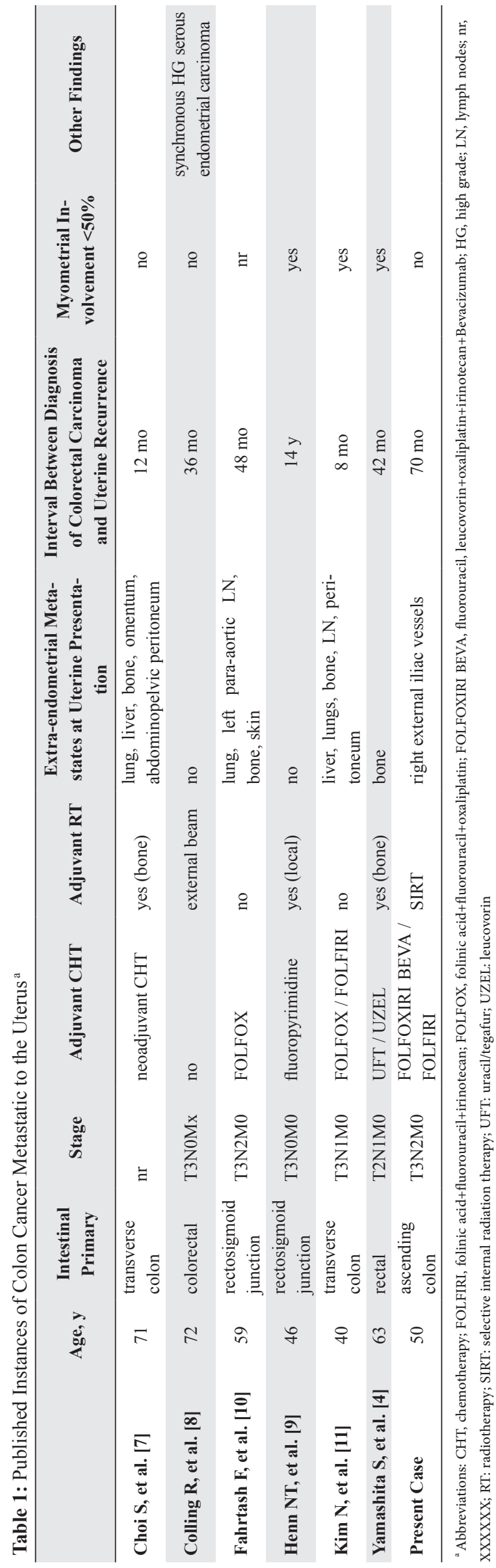

serosal tumor deposits should be present either macroscopically or microscopically, as well as the involvement of the outer third of the myometrial wall. Abu-Rustum et al., identified 3 cases of colonic metastasis in the uterus (endometrium or cervix) in their retrospective review over 18 years and concluded that adenocarcinoma arising during follow-up is more likely to be a primary endometrial adenocarcinoma than metastatic colon cancer to the endometrium [6].

Metastatic colonic adenocarcinoma may form large uterine tumor masses abutting into the uterine cavity and causing abnormal uterine bleeding, mimicking an endometrial carcinoma. Otherwise, the sparing of the endometrium accounts for the lack of symptoms, and uterine metastases are detected only by imaging studies. In our review of published cases (Table 1), all instances showing prevalent myometrial involvement $(>50 \%)$ were discarded, cause they could represent direct spread from colon cancer. Cases with ovarian metastases were also excluded as the endometrial involvement could have derived from intratubal or peritoneal seeding. The cases we selected are therefore believed to be embolic metastases reaching the endometrium through the hematogenous or lymphatic stream. Of the 6 cases presented in Table 1, only two showed no myometrial involvement [7, 8], and one had less than $50 \%$ myometrial involvement in the absence of other systemic spread [9]. The time interval between the initial intestinal presentation and the uterine recurrence ranged from 8 months to 14 years with a median period of 52 months. In the majority of cases, uterine metastases were found in the context of advanced metastatic disease.

Histologically, the differential diagnosis includes mucinous or endometrioid endometrial carcinoma. In the absence of relevant clinical history, some shared histological features among large bowel carcinoma and mucinous or endometrioid endometrial carcinoma may lead to a missed diagnosis. Clues to correct diagnosis are the excess of dirty necrosis in the tumor area, and the lack of dysplastic changes in the surrounding endometrium. Immunohistochemistry may be of confusion, as CDX2 may be expressed both in endometrioid and mucinous endometrial carcinoma, and ER can occasionally be expressed in colon cancers [12]. PAX8 may help exclude the Mullerian origin; while p16 immunostaining which may help to unmask 
an endocervical primary, was positive in our case. However, p16 protein is detectable in $80 \%$ of patients with colorectal carcinoma [13]; stressing the need for clinical-pathological correlations and awareness of the occurrence of the indirect spread of a large bowel carcinoma in the uterus.

In conclusion, metastases to the endometrium often pose diagnostic challenges for both pathologists and clinicians. The challenge is even more with metastatic colon tumors which can histologically present as a primary disease in the endometrium if there is little awareness of this condition and the pathologist is not alerted to consider metastasis. The clinical history of disseminated metastases, the lack of CK7 expression at immunohistochemistry, and positive results for CK20 and nuclear CDX2 support the diagnosis of metastatic carcinoma of colorectal origin.

\section{ACKNOWLEDGEMENTS}

None declared.

\section{CONFLICTS OF INTEREST}

The authors declared no conflict of interest.

\section{ETHICS APPROVAL}

This case was approved by the Institutional Review Board of the university hospital where it was performed. Patient provided written informed consent regarding the use of data for the case report.

\section{REFERENCES}

1. Kumar NB, Hart WR. Metastases to the uterine corpus from extragenital cancers. A clinicopathologic study of 63 cases. Cancer. 1982;50(10):2163-9. DOI: 10.1002/1097-0142(19821115)50:10<2163::aid-cncr2820501032>3.0.co;2-f PMID: 7127256.

2. MazurMT,HsuehS, GersellDJ.Metastasestothefemalegenital tract. Analysis of 325 cases. Cancer. 1984;53(9):1978-84. DOI: $10.1002 / 1097-0142(19840501) 53: 9<1978:$ :aid-cncr2820530929>3.0.co;2-1 PMID: 6322966.
3. Metser U, Haider MA, Khalili K, Boerner S. MR imaging findings and patterns of spread in secondary tumor involvement of the uterine body and cervix. AJR Am J Roentgenol. 2003;180(3):765-9. DOI: 10.2214/ajr.180.3.1800765 PMID: 12591694.

4. Yamashita S, Yuge A, Yano M, Kai K, Nasu K, Narahara H. Endometrial Metastasis from Primary Rectal Carcinoma: A Case Report and Literature Review. Austin J Obstet Gynecol. 2015;2(1):1032.

5. Weingold AB, Boltuch SM. Extragenital metastases to the uterus. Am J Obstet Gynecol. 1961;82:1267-72. DOI: 10.1016/s0002-9378(16)36252-4 PMID: 14005740.

6. Abu-Rustum N, Barakat RR, Curtin JP. Ovarian and uterine disease in women with colorectal cancer. Obstet Gynecol. 1997;89(1):85-7. DOI: 10.1016/s0029-7844(96)00369-9 PMID: 8990444.

7. Choi S, Joo JW, Do SI, Kim HS. Endometrium-Limited Metastasis of Extragenital Malignancies: A Challenge in the Diagnosis of Endometrial Curettage Specimens. Diagnostics (Basel). 2020;10(3). DOI: 10.3390/diagnostics10030150 PMID: 32164210.

8. Colling R, Lopes T, Das N, Mathew J. Endometrial metastasis of colorectal cancer with coincident endometrial adenocarcinoma. BMJ Case Rep. 2010;2010. DOI: 10.1136/ bcr.09.2009.2229 PMID: 22791861.

9. Henn NT, Zanin K, de Oliveira B, Schwingel D, Pastorello J. Uterine metastasis from the rectosigmoid transition cancer: a case report. J Histol Histopathol. 2017;4:6. DOI: 10.7243/2055-091X-4-6.

10. Fahrtash F, Chan D, Colebatch A, Rutovitz J. A very unusual presentation of metastatic colon cancer. ISRN Oncol. 2011;2011:531803. DOI: 10.5402/2011/531803 PMID: 22091421.

11. Kim N, Park SB, Lee JB, Park HJ, Kim MK, Hwang IG, et al. Imaging Findings of Endometrial Metastasis from Colon Cancer: A Case Report. J Korean Soc Radiol. 2013;69(4):301-5. DOI: 10.3348/jksr.2013.69.4.301.

12. Sciascia C, Olivero G, Comandone A, Festa T, Fiori MG, Enrichens F. Estrogen receptors in colorectal adenocarcinomas and in other large bowel diseases. Int J Biol Markers. 1990;5(1):38-42. DOI: $10.1177 / 172460089000500108$ PMID: 1700031 .

13. Lam AK, Ong K, Giv MJ, Ho YH. p16 expression in colorectal adenocarcinoma: marker of aggressiveness and morphological types. Pathology. 2008;40(6):580-5. DOI: 10.1080/00313020802320713 PMID: 18752124. 